\title{
Lattice Dynamics of Beryllium from a First-Principles Nonlocal Pseudopotential Approach
}

Walter, F. King; Cutler, P. H.

Published in:

Physical Review B

Link to article, DOI:

10.1103/PhysRevB.2.1733

Publication date:

1970

Document Version

Publisher's PDF, also known as Version of record

Link back to DTU Orbit

Citation (APA):

Walter, F. K., \& Cutler, P. H. (1970). Lattice Dynamics of Beryllium from a First-Principles Nonlocal

Pseudopotential Approach. Physical Review B, 2(6), 1733-1742. https://doi.org/10.1103/PhysRevB.2.1733

\section{General rights}

Copyright and moral rights for the publications made accessible in the public portal are retained by the authors and/or other copyright owners and it is a condition of accessing publications that users recognise and abide by the legal requirements associated with these rights.

- Users may download and print one copy of any publication from the public portal for the purpose of private study or research.

- You may not further distribute the material or use it for any profit-making activity or commercial gain

- You may freely distribute the URL identifying the publication in the public portal

If you believe that this document breaches copyright please contact us providing details, and we will remove access to the work immediately and investigate your claim. 


\title{
Lattice Dynamics of Beryllium from a First-Principles Nonlocal Pseudopotential Approach* ${ }^{\dagger}$
}

\author{
Walter F. King, III \\ Laboratory for Electrophysics, The Technical University, DK-2800 Lyngby, Denmark \\ and \\ P. H. Cutler \\ Department of Physics, The Pennsylvania State University, University Park, Pennsylvania 16802
}

(Received 15 April 1970)

\begin{abstract}
The lattice dynamics of beryllium, a metal with hexagonal close-packed structure and two atoms per unit cell, is investigated within the framework of Harrison's first-principles pseudopotential theory, using (i) the Slater approximation for the conduction-band-core exchange, and (ii) a modified dielectric-screening function employing the Kohn-Sham approximation for exchange among the conduction electrons. The energy-wave-number characteristic $F(q)$ is constructed from the Hartree-Fock-Slater (HFS) wave function for $\mathrm{Be}^{++}$; this is

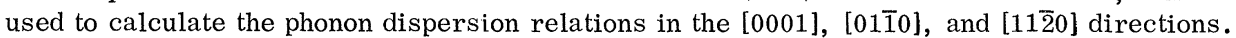
Good agreement is obtained with neutron diffraction experiments. The three independent elastic shear constants are also calculated from $F(q)$; good agreement with experiment is obtained for $C$ and $C^{\prime}$, but only fair results obtain for $c_{44}$.
\end{abstract}

\section{INTRODUCTION}

Until recently, most of the successful applications of first-principles pseudopotential theory to the calculation of phonon dispersion relations have been only with metals having one atom per unit cell. ${ }^{1,2}$ In this paper, the a priori pseudopotential theory based on the orthogonalized-plane-wave (OPW) method of Phillips and Kleinman ${ }^{3}$ and developed by Harrison ${ }^{4}$ for simple and polyvalent metals is extended and applied to beryllium, a metal with hexagonal close-packed (hcp) structure and two atoms per unit cell. Application to the lattice dynamics of magnesium is treated in a subsequent paper.

We have constructed a nonlocal pseudopotential from the Hartree-Fock-Slater (HFS) wave function ${ }^{5}$ for $\mathrm{Be}^{++}$and used it to calculate the phonon spectrum

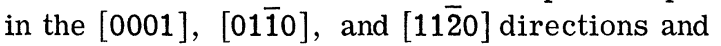
the elastic shear constants $C, C^{\prime}$, and $c_{44}$. Previously published dispersion relations for beryllium by Sahni et al. ${ }^{6}$ for a local pseudopotential and by Koppel and Maradudin ${ }^{7}$ for a nonlocal pseudopotential gave poor agreement with experiment. Roy and Venkataraman ${ }^{8}$ have calculated dispersion relations for magnesium, a metal also having hcp symmetry, using a first-principles nonlocal pseudopotential published by Harrison. ${ }^{1}$ Their results also show poor agreement between theory and experiment. One of the pitfalls in this type of calculation is exhibited in the papers by Roy and Venkataraman and Sahni et al.; this is the vanishing of the longitudinal acoustic (LA) branch at finite wave vectors.
When the wave vector is small, the relatively large electrostatic and band-structure components of the dynamical matrix nearly cancel each other and the small positive remainder is then used in the calculation of the LA frequency. Thus, a small error in the band-structure component may result in a negative remainder, which leads to an imaginary LA branch.

Sahni and Venkataraman ${ }^{9}$ have recently published results for the lattice dynamics of beryllium. They constructed both an a priori pseudopotential based upon the band-structure work of Loucks and Cutler ${ }^{10}$ and a model pseudopotential based upon an extension of the Heine-Abarenkov ${ }^{11}$ (HA) model potential. The phonon dispersion relations calculated from both of these approaches show generally poor agreement when compared to experimental data. Another recent calculation of the lattice dynamics of hcp metals by Gilat, Rizzi, and Cubiotti ${ }^{12}$ is based upon the HA method as reformulated and optimized by Shaw and Harrison. ${ }^{13}$ The beryllium phonon spectrum that one obtains from this method also compares poorly with the experimental results. An examination of the above papers indicates that calculations based upon first-principles theory have not produced very satisfactory phonon dispersion relations for the noncubic simple metals. The present work extends and expands upon a paper ${ }^{14}$ in which we reported on a successful first-principles nonlocal pseudopotential calculation of the phonon spectrum of an hcp metal.

Basic pseudopotential theory and its application to lattice dynamics has been discussed in many 
place ${ }^{15,16}$ and we shall restrict ourselves to a cursory description of Harrison's general theory and to points directly relevant to the calculation. The nonlocal pseudopotential theory developed by Harrison is based on a representation of the valence-electron wave functions by single orthogonalized plane waves (OPW's). When these are substituted into the one-electron Schrödinger equation, the terms can be rearranged to give a small effective interaction potential which, in theory, justifies the use of second-order perturbation theory to compute the conduction-band states.

The part of the total energy that is dependent upon the crystal structure can generally be divided into three contributions: (i) the Coulombic or Ewald term which arises from the electrostatic interaction of the ions, (ii) the band-structure energy which arises from the electron-ion-electron interaction, and (iii) the core-core repulsion or overlap term. The contribution from (i) was computed using the Ewald method ${ }^{17}$ for a lattice of point ions immersed in a uniform compensating background of negative charge, and that from (ii) was computed from Harrison's nonlocal pseudopotential method. ${ }^{16}$ We shall assume that the small core in beryllium makes the core overlap negligible and omit contribution (iii). Expressions for contributions (i) and (ii) are given in Sec. II along with a review of pertinent Born-von Kármán lattice dynamics and nonlocal pseudopotential theory.

In Sec. III we discuss the calculation of the crystal potential and application to the phonon dispersion relations and the elastic shear constants; the results and discussion are given in Sec. IV. Unless otherwise noted, all numerical values are given in atomic units where $\hbar=1, e^{2}=2$, the electronic mass equals $\frac{1}{2}$, and energy is in rydbergs. Table I contains the relevant physical parameters.

\section{THEORETICAL FORMULATION}

\section{A. Born-von Kármán Lattice Dynamics}

Let the position of a unit cell in a non-Bravais lattice be $\vec{a}(n)=n_{1} \vec{a}_{1}+n_{2} \vec{a}_{2}+n_{3} \vec{a}_{3}$, where the $\vec{a}_{i}$ are primitive lattice vectors and the $n_{i}$ are integers. We let $\overrightarrow{\mathrm{d}}_{j}$ identify the $j$ th atom in the unit cell. The reciprocal-lattice vectors $\vec{K}_{j}$ are defined from

TABLE I. Physical data for beryllium.

\begin{tabular}{lr}
\hline \hline Ionic mass & $m=8216$ \\
Atomic volume & $\Omega_{0}=54.75$ \\
Fermi wave vector & $k_{f}=1.026$ \\
Lattice constants & $a=4.3211$ \\
& $c=6.7716$ \\
Effective valence & $Z^{*}=2.1347$ \\
\hline \hline
\end{tabular}

the direct-lattice vectors in the usual manner and satisfy the condition

$$
\overrightarrow{\mathrm{a}}_{i} \cdot \overrightarrow{\mathrm{K}}_{j}=2 \pi \delta_{i j}
$$

Born-von Kármán lattice dynamics ${ }^{18}$ is based upon two approximations: (i) The potential energy of the crystalline solid is assumed to depend only upon the instantaneous nuclear coordinates, i. e., adiabatic approximation; (ii) when this potential energy is then expanded in powers of the amplitudes of the atomic vibrations and all terms higher than those which are quadratic in these amplitudes are neglected, the remaining terms constitute the harmonic approximation

$$
\Phi=\frac{1}{2} \sum_{n j \alpha} \sum_{n^{\prime} j^{\prime} \beta} \Phi_{\alpha \beta}\left(\begin{array}{c}
n n^{\prime} \\
j j^{\prime}
\end{array}\right) u_{\alpha}(n j) u_{\beta}\left(n^{\prime} j^{\prime}\right),
$$

where $u_{\alpha}(n j)$ is the alpha component of the displacement of the $j$ th atom in the $n$th unit cell and

$$
\Phi_{\alpha \beta}\left(\begin{array}{l}
n n^{\prime} \\
j j^{\prime}
\end{array}\right)=\left.\frac{\partial^{2} \Phi}{\partial u_{\alpha}(n j) \partial u_{\beta}\left(n^{\prime} j^{\prime}\right)}\right|_{0} .
$$

The subscript 0 means that the quantity is evaluated at equilibrium. When the equations of motion are solved in the standard manner the phonon frequencies are given by

$$
\begin{gathered}
\sum_{j^{\prime} \beta}\left[\left(m_{j} m_{j}\right)^{-1 / 2} D_{\alpha \beta}\left(\overrightarrow{\mathrm{Q}}, j j^{\prime}\right) U_{\beta}\left(\overrightarrow{\mathrm{Q}} j^{\prime}\right)\right. \\
\left.-\omega^{2}(\overrightarrow{\mathrm{Q}}) U_{\alpha}(\overrightarrow{\mathrm{Q}} j) \delta_{\alpha \beta} \delta_{j j^{\prime}}\right]=0
\end{gathered}
$$

where $\vec{Q}$ is the wave vector of the disturbance and the dynamical matrix can be defined as

$D_{\alpha \beta}\left(\overrightarrow{\mathrm{Q}}, j j^{\prime}\right)=\sum_{\overrightarrow{\mathrm{a}}} \Phi_{\alpha \beta}\left(\frac{\vec{\alpha}}{j j^{\prime}}\right) \exp \left[-i \overrightarrow{\mathrm{Q}} \cdot\left(\overrightarrow{\mathrm{a}}+\overrightarrow{\mathrm{d}}_{j^{\prime}}-\overrightarrow{\mathrm{d}}_{j}\right)\right]$.

The $m_{j}$ are the ionic masses. To simplify the notation, we have suppressed the triplet $\left(n_{1} n_{2} n_{3}\right)$ and have simply written $\vec{a}$ for the direct-lattice vectors. The condition that the set of equations (1) have a solution is that the determinant of the coefficients vanish:

$$
\operatorname{det}\left(\underline{D}-\omega^{2} m \delta_{j j}, \delta_{\alpha \beta}\right)=0
$$

\section{B. Nonlocal Pseudopotential Formalism}

Three basic approximations are necessary in Harrison's formulation of the theory. These are (i) the use of a self-consistent field which represents some average interaction, (ii) the small-core approximation which allows the core and conduction-band states to be separated, and (iii) the use of perturbation theory to calculate conduction-band states and energies. These approximations are appropriate for the alkali and polyvalent metals but are generally unsuitable for the noble and transition metals. The theory is based upon a representation of the valence states by single OPW's. 
In the core region the oscillatory behavior of the valence-electron wave functions can be interpreted as deriving from the orthogonality requirement between the core and valence states. This can be described by a repulsive potential $V_{R}$ which almost cancels the crystal potential $V$ and allows one to represent the core-valence interaction by the weak resultant potential called the pseudopotential:

$$
W=V+V_{R}
$$

The pseudopotential equation is obtained directly from the one-electron Schrödinger equation and has the form

$$
(T+W) \phi_{k}=E_{k} \phi_{k},
$$

with

$$
W=V+\sum_{\alpha}\left(E_{k}-E_{\alpha}\right)|\alpha\rangle\langle\alpha|,
$$

where the $\phi_{k}$ are the pseudo-wave-functions, the $|\alpha\rangle$ are core functions, and the $E_{\alpha}$ are the core energies in the metal. The $E_{\alpha}$ can be obtained from the core energies in the free ion by applying a correction that Lin and Phillips ${ }^{19}$ have termed a core shift. The magnitude of this shift is critical to the calculation, although its determination is somewhat arbitrary. We shall discuss its evaluation in a later section.

The band-structure contribution to the total energy can be obtained from the second-order term in the usual perturbation expansion for $E_{k}$. As the Coulomb interaction between any pair of electrons has been counted twice, the electron-electron interaction must be subtracted from this to obtain the correct energy. When the lattice-dependent factors are collected in a structure factor $S(\overrightarrow{\mathrm{q}})$, the result may be written as

$$
E_{\mathrm{bs}}=\sum_{\overrightarrow{\mathrm{q}}}^{\prime} S^{*}(\overrightarrow{\mathrm{q}}) S(\overrightarrow{\mathrm{q}}) F(q),
$$

where $F(q)$ is the transform of the ion-electron-ion potential and is called the energy-wave-number characteristic. It is a function of the atomic volume as well as of the local potentials but is independent of the structural configuration. Detailed expressions for the calculation of $F(q)$ may be found in Harrison's book. ${ }^{20}$

The use of single OPW's to approximate the conduction-electron wave functions gives rise to an additional charge which moves rigidly with the ions. This charge, which was derived by Pick and Sarma ${ }^{21}$ in their reformulation of Harrison's approach, can be combined with the valence charge to obtain an effective valence $Z^{*}$ which is larger than the valence.

\section{Dynamical Matrix}

The Coulombic contribution to the dynamical matrix can be derived by methods due to Ewald ${ }^{17}$ and Thompson. ${ }^{22,23} \mathrm{~A}$ more modern and rather elegant derivation of this matrix has been given by Cochran. ${ }^{24}$ We shall not attempt to duplicate these rather long and tedious derivations but will merely give the results:

$$
\begin{aligned}
B_{\alpha \beta}\left(\overrightarrow{\mathrm{Q}}, j j^{\prime}\right)= & \sum_{\mathbf{a}} \cdot\left[\frac { ( \vec { \mathrm { a } } + \vec { \mathrm { d } } _ { j ^ { \prime } } - \vec { \mathrm { d } } _ { j ^ { \prime } } ) _ { \alpha } ( \vec { \mathrm { a } } + \vec { \mathrm { d } } _ { j ^ { \prime } } - \vec { \mathrm { d } } _ { j ^ { \prime } } ) _ { \beta } } { | \vec { \mathrm { a } } + \vec { \mathrm { d } } _ { j ^ { \prime } } - \vec { \mathrm { d } } _ { j } | ^ { 2 } } \left(\frac{3 \operatorname{erfc}\left(\eta \mid \overrightarrow{\mathrm{a}}+\overrightarrow{\mathrm{d}}_{j^{\prime}}-\overrightarrow{\mathrm{d}}_{j}{ }^{\prime}\right)}{\left|\overrightarrow{\mathrm{a}}+\overrightarrow{\mathrm{d}}_{j^{\prime}}-\overrightarrow{\mathrm{d}}_{j^{\prime}}\right|^{2}}+\frac{6 \eta}{\sqrt{\pi}} \frac{\exp \left(-\eta^{2}\left|\overrightarrow{\mathrm{a}}+\overrightarrow{\mathrm{d}}_{j^{\prime}}-\overrightarrow{\mathrm{d}}_{j^{\prime}}\right|^{2}\right)}{\left|\overrightarrow{\mathrm{a}}+\overrightarrow{\mathrm{d}}_{j^{\prime}}-\overrightarrow{\mathrm{d}}_{j}\right|^{2}}\right.\right. \\
& \left.\left.+\frac{4 \eta^{3}}{\sqrt{\pi}} \exp \left(-\eta^{2}\left|\overrightarrow{\mathrm{a}}+\overrightarrow{\mathrm{d}}_{j^{\prime}}-\overrightarrow{\mathrm{d}}_{j}\right|^{2}\right)\right)-\left(\frac{\operatorname{erfc}\left(\eta\left|\overrightarrow{\mathrm{a}}+\overrightarrow{\mathrm{d}}_{j^{\prime}}-\overrightarrow{\mathrm{d}}_{j}\right|\right)}{\left|\overrightarrow{\mathrm{a}}+\overrightarrow{\mathrm{d}}_{j^{\prime}}-\overrightarrow{\mathrm{d}}_{j}\right|^{3}}+\frac{2 \eta}{\sqrt{\pi}} \frac{\exp \left(-\eta^{2}\left|\overrightarrow{\mathrm{a}}+\overrightarrow{\mathrm{d}}_{j^{\prime}}-d_{j}\right|^{2}\right)}{\left|\overrightarrow{\mathrm{a}}+\overrightarrow{\mathrm{d}}_{j^{\prime}}-\overrightarrow{\mathrm{d}}_{j}\right|^{2}}\right) \delta_{\alpha \beta}\right] \\
& \times \exp \left[i \overrightarrow{\mathrm{Q}} \cdot\left(\overrightarrow{\mathrm{a}}+\overrightarrow{\mathrm{d}}_{j^{\prime}}-\overrightarrow{\mathrm{d}}_{j}\right)\right], \\
A_{\alpha \beta}\left(\overrightarrow{\mathrm{Q}}, j j^{\prime}\right)= & \frac{4 \pi}{s \Omega_{0}}\left(\sum_{\overrightarrow{\mathrm{K}}} \frac{(\overrightarrow{\mathrm{K}}+\overrightarrow{\mathrm{Q}})_{\alpha}(\overrightarrow{\mathrm{K}}+\overrightarrow{\mathrm{Q}})_{\beta}}{|\overrightarrow{\mathrm{K}}+\overrightarrow{\mathrm{Q}}|^{2}}\right) \exp \left(-|\overrightarrow{\mathrm{K}}+\overrightarrow{\mathrm{Q}}|^{2} / 4 \eta^{2}\right) \exp \left[i \overrightarrow{\mathrm{K}} \cdot\left(\overrightarrow{\mathrm{d}}_{j}-\overrightarrow{\mathrm{d}}_{j^{\prime}}\right)\right],
\end{aligned}
$$

where $s$ is the number of atoms in a unit cell and $\Omega_{0}$ is the atomic volume. The prime over the $\vec{a}$ sum means that the denominator is never zero. The dynamical matrix is then

$$
\begin{aligned}
D_{\alpha \beta}^{c}\left(\overrightarrow{\mathrm{Q}}, j j^{\prime}\right)= & Z_{j}^{2} A_{\alpha \beta}\left(\overrightarrow{\mathrm{Q}}, j j^{\prime}\right)-Z_{j}^{2} B_{\alpha \beta}\left(\overrightarrow{\mathrm{Q}}, j j^{\prime}\right), j \neq j^{\prime} \\
D_{\alpha \beta}^{c}(\overrightarrow{\mathrm{Q}}, j j)= & Z_{j}^{2}\left\{A_{\alpha \beta}(\overrightarrow{\mathrm{Q}}, j j)-B_{\alpha \beta}(\overrightarrow{\mathrm{Q}}, j j)\right. \\
& \left.+\sum_{j^{\prime}}^{\prime}\left[B_{\alpha \beta}\left(0, j j^{\prime}\right)-A_{\alpha \beta}\left(0, j j^{\prime}\right)\right]\right\}, \quad j=j^{\prime} .
\end{aligned}
$$

The parameter $\eta$ is chosen to make both the sums in direct and reciprocal space converge rapidly; $\operatorname{erfc}(x)$ is the complementary error function. Good results can be obtained by choosing $\eta=\left(4 / \Omega_{0}\right)^{1 / 3}$.
When a periodic distortion is introduced into the lattice, the accompanying change in band-structure energy can be evaluated by expanding the structure factors to second order in the amplitudes of the disturbance and subtracting the equilibrium energy:

$$
\delta E_{\mathrm{bs}}=E_{\mathrm{bs}} \text { (distorted) }-E_{\mathrm{bs}} \text { (equilibrium). }
$$

The electronic component of the dynamical matrix can then be written by comparing Eq. (2) to the general expression for the crystal potential energy in the harmonic approximation. This derivation is discussed by Harrison, ${ }^{25}$ and Roy and Venkataraman. ${ }^{8}$ The resulting expression is 


$$
\begin{aligned}
D_{\alpha \beta}^{E}\left(\overrightarrow{\mathrm{Q}}, j j^{\prime}\right)= & \sum_{\overrightarrow{\mathrm{K}}}(\overrightarrow{\mathrm{K}}+\overrightarrow{\mathrm{Q}})_{\alpha}(\overrightarrow{\mathrm{K}}+\overrightarrow{\mathrm{Q}})_{\beta} \\
& \times F(|\overrightarrow{\mathrm{K}}+\overrightarrow{\mathrm{Q}}|) \exp \left[i \overrightarrow{\mathrm{K}} \cdot\left(\overrightarrow{\mathrm{d}}_{j}-\overrightarrow{\mathrm{d}}_{j^{\prime}}\right)\right], \\
D_{\alpha \beta}^{E}(\overrightarrow{\mathrm{Q}}, j j)= & \sum_{\overrightarrow{\mathrm{K}}}\left[(\overrightarrow{\mathrm{K}}+\overrightarrow{\mathrm{Q}})_{\alpha}(\overrightarrow{\mathrm{K}}+\overrightarrow{\mathrm{Q}})_{\beta} F(|\overrightarrow{\mathrm{K}}+\overrightarrow{\mathrm{Q}}|)\right. \\
& \left.-K_{\alpha} K_{\beta} \xi(j) F(K)\right],
\end{aligned}
$$

where

$$
\xi(j)=\sum_{j^{\prime}} \cos \left[\overrightarrow{\mathrm{K}} \cdot\left(\overrightarrow{\mathrm{d}}_{j}-\overrightarrow{\mathrm{d}}_{j^{\prime}}\right)\right] .
$$

When $K=0$, the term involving $F(K)$ is omitted.

\section{CALCULATION OF CRYSTAL POTENTIAL AND CORE SHIFT}

\section{A. Crystal Potential}

In the self-consistent-field approximation, one assumes that all electrons see the same average potential. The interactions that contribute to this potential $\operatorname{are}^{20}$ (i) the potential due to the ion core, (ii) the conduction-band-core exchange, (iii) the correlation between conduction and core electrons, (iv) the potential due to the conduction electrons, (v) the screening potential, and (vi) the exchange among conduction electrons.

\section{Coulomb Potential due to Ion Cores}

The wave functions for the core electrons in the simple metals do not vary much from the one-electron wave functions for the free ions or from the atomic wave functions. We used the $1 s$ HartreeFock-Slater (HFS) wave function for the free beryllium ion $\mathrm{Be}^{++}$since a program to generate these wave functions for all of the elements has been written by Herman and Skillman. ${ }^{5}$ The electron density $\rho(r)$ was constructed from this wave function and, with the aid of Poisson's equation, was transformed to $q$ space to give a potential contribution

$$
v^{(1 e)}(q)=\left(4 \pi e^{2} / \Omega_{0} q^{2}\right) n(q),
$$

where the $q$-space electron density is

$$
n(q)=\int \rho(r) \exp (-i \overrightarrow{\mathrm{q}} \cdot \overrightarrow{\mathrm{r}}) d^{3} r .
$$

This density is defined such that $n(0)$ equals the number of core electrons. The nuclear-charge contribution to the potential is simply

$$
v^{(1 n)}(q)=-\left(4 \pi e^{2} / \Omega_{0} q^{2}\right) A,
$$

where $A$ is the atomic number. The total ion-core contribution is then

$$
v^{(1)}(q)=v^{(1 n)}(q)+v^{(1 e)}(q) .
$$

This potential is tabulated in Table II, column 2, for selected values of $q / k_{F}$.

\section{Conduction-Band-Core Exchange}

We first assumed that the valence electrons could be represented by the $2 s$ atomic wave functions in
TABLE II. Fourier components of the potentials.

\begin{tabular}{rrrrr}
\hline \hline$q / k_{F}$ & \multicolumn{1}{c}{$-v_{q}^{(1)}$} & $-v_{q}^{(2)}$ & $-v_{q}^{(3)}$ & $-v_{q}^{(4)}$ \\
\hline 0.00 & $\ldots$ & 0.2779 & 0.0015 & $\ldots$. \\
0.01 & 8713.4561 & 0.3061 & 0.0015 & 586.9297 \\
0.05 & 348.5706 & 0.3058 & 0.0015 & 23.4750 \\
0.10 & 87.1679 & 0.3049 & 0.0015 & 5.8671 \\
0.20 & 21.8172 & 0.3014 & 0.0015 & 1.4651 \\
0.30 & 9.7152 & 0.2956 & 0.0015 & 0.6499 \\
0.40 & 5.4795 & 0.2878 & 0.0015 & 0.3646 \\
0.50 & 3.5188 & 0.2783 & 0.0015 & 0.2325 \\
0.60 & 2.4538 & 0.2673 & 0.0015 & 0.1608 \\
0.70 & 1.8115 & 0.2551 & 0.0014 & 0.1175 \\
0.80 & 1.3946 & 0.2421 & 0.0014 & 0.0895 \\
0.90 & 1.1087 & 0.2286 & 0.0014 & 0.0702 \\
1.00 & 0.9041 & 0.2148 & 0.0014 & 0.0565 \\
1.10 & 0.7527 & 0.2010 & 0.0013 & 0.0463 \\
1.20 & 0.6374 & 0.1874 & 0.0013 & 0.0386 \\
1.30 & 0.5477 & 0.1742 & 0.0013 & 0.0326 \\
1.40 & 0.4764 & 0.1615 & 0.0012 & 0.0278 \\
1.50 & 0.4188 & 0.1494 & 0.0012 & 0.0240 \\
1.60 & 0.3717 & 0.1379 & 0.0012 & 0.0208 \\
1.70 & 0.3325 & 0.1272 & 0.0011 & 0.0182 \\
1.80 & 0.2996 & 0.1171 & 0.0011 & 0.0161 \\
1.90 & 0.2717 & 0.1077 & 0.0011 & 0.0142 \\
2.00 & 0.2479 & 0.0991 & 0.0010 & 0.0127 \\
2.20 & 0.2094 & 0.0837 & 0.0009 & 0.0102 \\
2.40 & 0.1799 & 0.0707 & 0.0009 & 0.0083 \\
2.60 & 0.1568 & 0.0598 & 0.0008 & 0.0068 \\
2.80 & 0.1383 & 0.0506 & 0.0007 & 0.0057 \\
3.00 & 0.1232 & 0.0430 & 0.0006 & 0.0048 \\
3.50 & 0.0955 & 0.0290 & 0.0004 & 0.0032 \\
4.00 & 0.0769 & 0.0200 & 0.0003 & 0.0022 \\
5.00 & 0.0536 & 0.0102 & 0.0001 & 0.0011 \\
6.00 & 0.0398 & 0.0056 & 0.0000 & 0.0006 \\
7.00 & 0.0307 & 0.0033 & $\ldots$ & 0.0003 \\
8.00 & 0.0244 & 0.0021 & $\ldots$ & 0.0002 \\
9.00 & 0.0198 & 0.0014 & $\ldots .$. & 0.0001 \\
10.00 & 0.0163 & 0.0009 & $\ldots$ & 0.0001 \\
\hline \hline & & & & \\
\hline
\end{tabular}

the core region and used a method described by Hartree ${ }^{26}$ to construct the Hartree-Fock (HF) effective potential for the exchange interaction between the core and conduction electrons. When this potential was used in the calculation, the resulting dispersion curves were found to be as much as $50 \%$ above the experimental values. We then computed an effective-exchange potential from the KohnSham ${ }^{27}$ approximation which is two-thirds of the Slater ${ }^{28}$ exchange. The results were very similar to those obtained from the $\mathrm{HF}$ effective potential. The best results for beryllium were obtained from the simple Slater approximation

$$
v^{(2)}(r)=-\left(3 e^{2} / 2 \pi\right)\left[3 \pi^{2} \rho(r)\right]^{1 / 3} .
$$

This potential is tabulated in column 3 of Table II. 


\section{Correlation between Conduction and Core Electrons}

Since it is well known ${ }^{16}$ that this contribution is relatively small when compared to the other terms in the crystal potential, we have used the following approximation scheme to calculate the conductioncore-electron correlations. We have assumed that for the first approximation, the correlation between the conduction and core electrons is the same as that for the free-electron gas. When one electron is added to the system of density $n$, the increase in the correlation energy is the effective-correlation potential $v_{c l}$. Then, if $\epsilon$ is the average correlation energy per particle, ${ }^{29}$ we obtain

$$
v_{c l}=\frac{d(n \epsilon)}{d n}
$$

For metallic densities Pines ${ }^{30}$ gives

$$
\epsilon \simeq 0.031 \ln r_{s}-0.115 \mathrm{Ry},
$$

where $r_{s}=(3 / 4 \pi n)^{1 / 3}$. To ensure that $v_{c l}(r) \rightarrow 0$ as $r \rightarrow \infty$, it is necessary to include the constant-valence charge density in the calculation. This latter term may then be subtracted at the end of the calculation. This potential is listed in column 4 of Table II.

\section{Potential due to Conduction Electrons}

We follow Harrison ${ }^{20}$ and define this potential to be due to the charge density of single-OPW states. In the probability density for a normalized OPW, the nonconstant terms give a localized electron density at each ion site which when summed over all occupied states results in a positive charge distribution. Harrison calls this the "orthogonalization hole" and combines it with the valence charge to form an effective valence $Z^{*}$. This orthogonalization hole contains $\left(Z-Z^{*}\right)$ electrons. If we assume that the hole distribution function is similar to that of the core electrons, we obtain

$$
v^{(4)}(q)=-\left[4 \pi e^{2} / \Omega_{0} q^{2} n(0)\right]\left(Z^{*}-Z\right) n(q) .
$$

This potential is tabulated in column 5 of Table II.

\section{Screening Potential}

This is the change in potential obtained when single OPW's are replaced by multiple OPW's in the valence states. This is treated by Harrison ${ }^{16}$ who concludes that (i) the change is of first order but the matrix elements enter the energy squared and (ii) the calculation of the screening field is justified in the plane-wave approximation.

\section{Exchange among Conduction Electrons}

We follow Cohen and Phillips, ${ }^{31}$ and Harrison ${ }^{20}$ and assume that a fluctuation in the electron density will cause an exchange potential $X_{q}$ in addition to the direct-interaction potential. The Fourier component for the total potential seen by an electron is then

$$
W_{q}^{1}=\left(4 \pi e^{2} / q^{2}+X_{q}\right) n_{q},
$$

where $n_{q}$ is the transform of the valence-electron density. The form of the potential that we have used is based on a suggestion by Harrison ${ }^{20}$ and utilizes the Kohn-Sham ${ }^{27}$ effective-exchange potential

$$
E_{x}=\frac{d\left(n \epsilon_{x}\right)}{d n}
$$

where $\epsilon_{x}$ is the exchange energy per electron of a uniform gas of density $n$. At $q=0, X_{0}$ should equal the exchange potential for a uniform electron gas at that density, which is given by

$$
X_{0}=\frac{d E_{x}}{d n}=\frac{-e^{2} \pi}{k_{F}^{2}},
$$

where $k_{F}$ is the Fermi wave vector. At large values of $q$, we use Hubbard's ${ }^{32}$ result that the ratio of the exchange to the Coulombic potential approaches $\frac{1}{2}$. This gives $X_{q}=-2 \pi e^{2} / q^{2}$ at large $q$. An expression for $X_{q}$ that will exhibit these asymptotic values is

$$
X_{q}=-\frac{e^{2} \pi}{k_{F}^{2}}\left(\frac{1}{1+q^{2} /\left(2 k_{F}^{2}\right)}\right) .
$$

When Eq. (3) is used to calculate the screened pseudopotential in terms of the unscreened elements, the dielectric function that obtains is

$$
\epsilon^{*}(q)=1+\left[1+\left(q^{2} X_{q} / 4 \pi e^{2}\right)\right][\epsilon(q)-1],
$$

where $\epsilon(q)$ is the static dielectric function. The justification for the use of this dielectric function or the slightly modified version of it lies in the relatively low phonon frequencies as compared with the electronic frequencies. The screening action of the electron gas is similar to that of a static external charge distribution. ${ }^{33}$ Further justification for this dielectric function can be found in an investigation made by Koppel. ${ }^{34}$ He has shown that for beryllium, the off-diagonal elements of the dielectric screening matrix are approximately two orders of magnitudes smaller than the corresponding diagonal elements. As can be seen in Fig. 1, there is little difference between the use of the modified and the free-electron dielectric function.

\section{B. Core Shift}

In the calculation of the screened matrix elements, the core eigenvalues $E_{\alpha}$ always appear together with $\langle\overrightarrow{\mathrm{k}}|V| \overrightarrow{\mathrm{k}}\rangle$ as $V_{\alpha}=\langle\overrightarrow{\mathrm{k}}|V| \overrightarrow{\mathrm{k}}\rangle-E_{\alpha}$, where the subscript $\alpha$ represents the quantum numbers $n$ and $l$. The use of the rigid-ion approximation means we assume the core eigenvalues $E_{\alpha}$ are shifted from their freeion values but that the core wave functions are not modified. The zero-order approximation to the $E_{\alpha}$ are, thus, the HFS energy eigenvalues, and the 


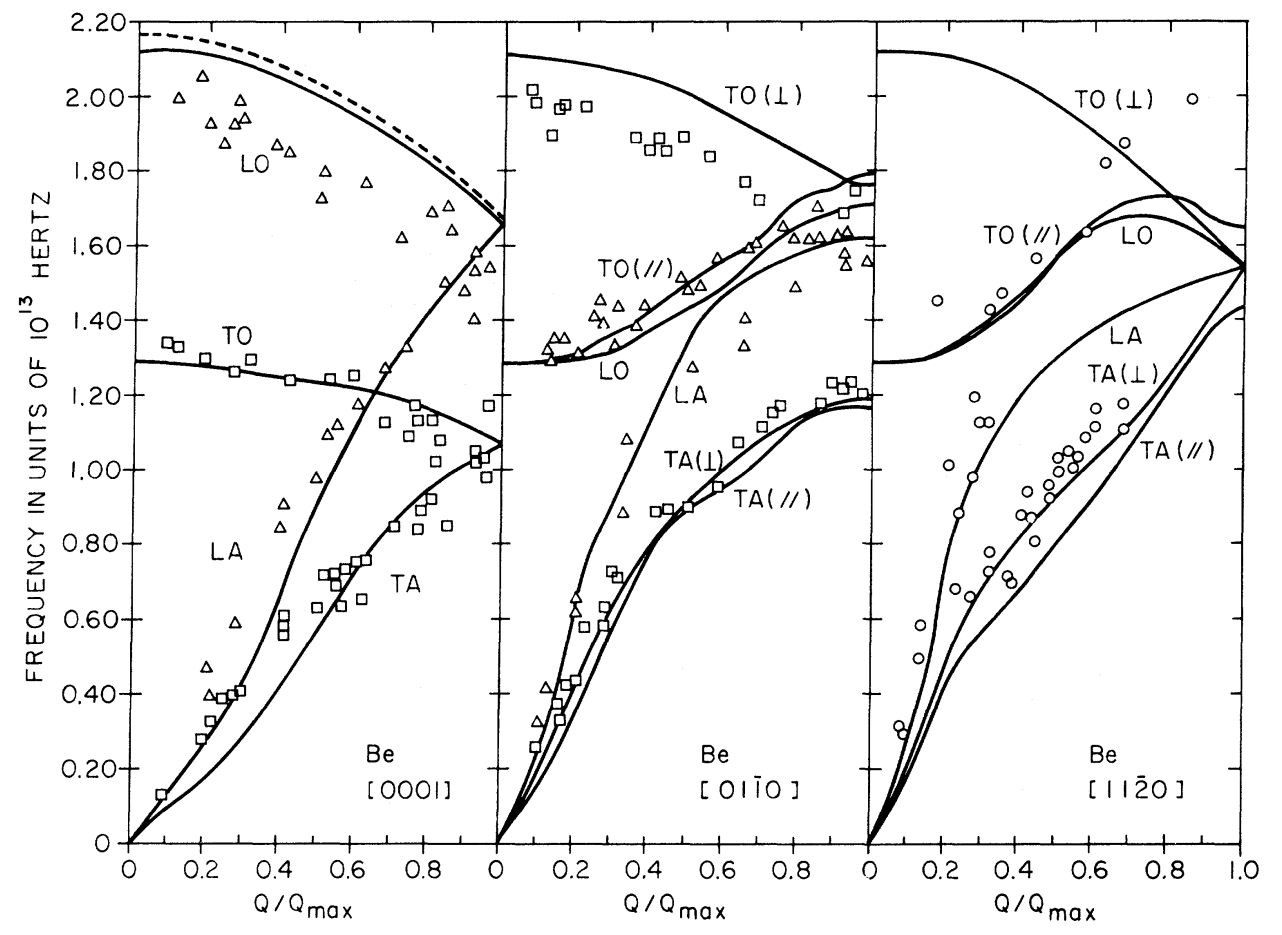

FIG. 1. Theoretical dispersion curves for the three crystallographic directions [0001], [011̄0], and [11 20$]$ in beryllium. The pseudopotential for these calculations was constructed from the Hartree-FockSlater wave function (Ref. 5) for $\mathrm{Be}^{++}$. In the [0001] direction the curves were computed with both the free-electron and the modified dielectric function. The results are almost identical with the exception of the LO branch, which is drawn as a dashed line for the free-electron case. The experimental points are from Schmunk (Ref. 39).

shift in energy is called the orthogonalization hole potential $^{20} v_{\mathrm{OPW}}$. The contributions to the potential at $q=0$ are all easily calculated with the exception of $v_{\mathrm{OPW}}$. Harrison originally approximated this potential by its value at the nucleus but, since this is clearly its maximum value, he suggests that a weighted average of $v_{\text {OPW }}$ might be more appropriate. ${ }^{20}$ If we define the average value of this potential as $v_{\mathrm{OPW}}^{*}=\alpha v_{\mathrm{OPW}}$, where $v_{\mathrm{OPW}}$ is the value at the nucleus, then $\alpha$ has a value of 0.625 when a distribution of the form $e^{-\mu r}$ is assumed. This is obviously still only a crude approximation since the value depends upon the form of the distribution that we assume for the orthogonalization hole. It seems, however, to be a better estimate than to take the maximum value, so we have used $\alpha=0.625$ in the present calculations.

In the computation of $v_{\text {OP }}$, we follow Harrison ${ }^{20}$ and assume that the orthogonalization hole due to each core shell has the same distribution function as the charge density for that core shell. Then the potential at the nucleus due to a shell is

$$
v_{n l}=2(2 l+1) \int\left[P_{n l}^{2}(r) / r\right] d r,
$$

where the $P_{n l}(r)$ are the radial wave functions.

The value of $v_{\mathrm{OPW}}$ at the nucleus is then

$$
v_{\mathrm{OPW}}=-\sum_{n l} v_{n l}\left(2 \Omega_{0} / \pi^{2}\right) \int_{0}^{k_{F}} k^{2}|\langle n l \mid \overrightarrow{\mathrm{k}}\rangle|^{2} d k,
$$

where the $\langle n l \mid \overrightarrow{\mathrm{k}}\rangle$ are the orthogonality coefficients. Using Harrison' $\mathrm{s}^{20}$ approximation for $v^{(1)}(0)$, we can write $V_{\alpha}$ explicitly as

$$
V_{n l}=v^{(1)}(0)+v^{(2)}(0)+v^{(3)}(0)-v_{\mathrm{OPW}}^{*}+\left|\epsilon_{n l}\right| .
$$

The $\epsilon_{n l}$ are the HFS energy eigenvalues which, for $\mathrm{Be}^{++}$, consist only of the $1 s$ value. These quantities are listed in Table III.

\section{Elastic Constants}

In hexagonal crystals, there are five independent elastic constants, three of which are pure shears that conserve the volume. ${ }^{35}$ The usual notation defining these shear constants is

$$
\begin{aligned}
& \text { (i) } C^{\prime}=\frac{1}{2}\left(c_{11}-c_{12}\right), \\
& \text { (ii) } c_{44}, \\
& \text { (iii) } C=c_{11}+c_{12}+2 c_{33}-4 c_{13} .
\end{aligned}
$$

Constant (i) derives from a shear that alters the angle between the axes in the basal plane, (ii) results from a bending of the hexagonal axis with respect to the basal plane, and (iii) results from a

TABLE III. Values for the calculation of $V_{\alpha}$.

\begin{tabular}{lc}
\hline$v^{(1)}(0)$ & -3.2962 \\
$v^{(2)}(0)$ & -0.2779 \\
$v^{(3)}(0)$ & -0.0015 \\
$\alpha$ & 0.625 \\
$\epsilon_{1 s}$ & -10.6086 \\
$v_{\text {opw }}$ & -1.90603 \\
$v_{\text {opw }}^{*}$ & -1.1913 \\
$V_{10}$ & 8.2243 \\
\hline
\end{tabular}


strain that alters the $c / a$ ratio. A detailed discussion of the elastic-shear constants of hexagonal metals is given by Cousins. ${ }^{36}$

A hcp structure can be considered to be two simple interpenetrating hexagonal lattices. When one of them is displaced relative to the other, there is internal strain. Born and Huang ${ }^{18}$ have investigated the effects of internal strain on the second-order elastic constants for nonmetals and have shown that the neglect of this strain leads to very small errors for central-force interactions. Cousins ${ }^{36}$ relates this work to metals and concludes that the omission of internal strains in the calculation of the secondorder elastic constants will not lead to errors larger than $6 \%$. We, therefore, also neglect these strains.

In the region where Hooke's law is valid, the strain energy of a hexagonal crystal may be written as

$$
\begin{aligned}
E= & E^{0}+\frac{1}{2} \Omega_{0}\left[c_{11}\left(s_{1}^{2}+s_{2}^{2}\right)+c_{33} s_{3}^{2}+2 c_{12} s_{1} s_{2}\right. \\
& \left.+2 c_{13}\left(s_{1} s_{3}+s_{2} s_{3}\right)+c_{44}\left(s_{4}^{2}+s_{5}^{2}\right)+\frac{1}{2}\left(c_{11}-c_{12}\right) s_{6}^{2}\right],
\end{aligned}
$$

where $E^{0}$ is the equilibrium energy, $s_{1}, s_{2}$, and $s_{3}$ are normal strains, and $s_{4}, s_{5}$, and $s_{6}$ are angular decreases in the $y z$, the $x z$, and the $x y$ angles, respectively. We shall take the $z$ axis in the [0001] direction, i.e., along the $c$ axis.

In the calculation of the second derivatives of the strain energy of the crystal, it is customary to write the direct- and reciprocal-lattice vectors in terms of strain parameters $\eta, \gamma_{1}$, or $\gamma_{2}$. For the constant $C$ these are ${ }^{35,37}$ for the direct-lattice vectors

$$
\begin{aligned}
& \overrightarrow{\mathrm{a}}_{1}=c(1+\eta) \hat{e}_{z}, \\
& \overrightarrow{\mathrm{a}}_{2}=a(1+\eta)^{-1 / 2} \hat{e}_{x}, \\
& \overrightarrow{\mathrm{a}}_{3}=a(1+\eta)^{-1 / 2}\left(-\frac{1}{2} \hat{e}_{x}+\frac{1}{2} \sqrt{3} \hat{e}_{y}\right),
\end{aligned}
$$

and for the reciprocal-lattice vectors

$$
\begin{aligned}
& \overrightarrow{\mathrm{K}}_{1}=(2 \pi / c)(1+\eta)^{-1} \hat{e}_{z}, \\
& \overrightarrow{\mathrm{K}}_{2}=(2 \pi / a)\left[(1+\eta)^{1 / 2} \hat{e}_{x}+(1 / \sqrt{3})(1+\eta)^{1 / 2} \hat{e}_{y}\right], \\
& \overrightarrow{\mathrm{K}}_{3}=(4 \pi / a \sqrt{3})(1+\eta)^{1 / 2} \hat{e}_{y} .
\end{aligned}
$$

The second derivative of the strain energy then gives

$$
\frac{d^{2} E}{d \eta^{2}}=\frac{1}{2} C \Omega_{0}
$$

at the equilibrium condition $\eta=0$.

For $C^{\prime}$ and $c_{44}$ the direct-lattice vectors are $\mathrm{ar}^{35}$

$$
\begin{aligned}
& \overrightarrow{\mathrm{a}}_{1}=c \hat{e}_{z}, \\
& \overrightarrow{\mathrm{a}}_{2}=a \gamma_{1}^{1 / 2} \hat{e}_{x}, \\
& \overrightarrow{\mathrm{a}}_{3}=\frac{1}{2} a \gamma_{1}^{1 / 2}\left(-\hat{e}_{x}+\gamma_{1}^{-1} \sqrt{3} \hat{e}_{y}\right),
\end{aligned}
$$

with

$$
\frac{d^{2} E}{d \gamma_{1}^{2}}=C^{\prime} \Omega_{0} \text { at } \gamma_{1}=1
$$

and

$$
\begin{aligned}
& \overrightarrow{\mathrm{a}}_{1}=c \hat{e}_{z}, \\
& \overrightarrow{\mathrm{a}}_{2}=a\left(\hat{e}_{x}+\gamma_{2} \hat{e}_{z}\right), \\
& \overrightarrow{\mathrm{a}}_{3}=\frac{1}{2} a\left(-\hat{e}_{x}+\sqrt{3} \hat{e}_{y}-\gamma_{2} \hat{e}_{z}\right),
\end{aligned}
$$

with

$$
\frac{d^{2} E}{d \gamma_{2}^{2}}=c_{44} \Omega_{0} \text { at } \gamma_{2}=0 \text {. }
$$

The total crystal energy can be separated into four parts: (i) the free-electron energy which depends only upon the volume and not on the ionic positions, (ii) the electrostatic energy caused by the positive point charges immersed in a uniform compensating negative charge background, (iii) the repulsive energy of the ion-core interactions, and (iv) the band-structure energy. The free-electron energy may be neglected in any calculation of shear constants since it depends only upon the volume. For the small ion cores of beryllium, we assume that the core repulsion is negligible, which leaves only the electrostatic and band-structure energies to be considered. The electrostatic energy can be calculated by Ewald's method ${ }^{17,38}$ and the band-structure energy is given by the expression for $E_{\mathrm{bs}}$ in Sec. II B:

$$
E_{\mathrm{bs}}=\sum_{\overrightarrow{\mathrm{d}}}^{\prime} S(\overrightarrow{\mathrm{q}}) S(\overrightarrow{\mathrm{q}}) F(q)=\sum_{\overrightarrow{\mathrm{K}}}^{\prime} \cos ^{2}\left(\frac{1}{2} \overrightarrow{\mathrm{K}} \cdot \overrightarrow{\mathrm{d}}_{2}\right) F(K) .
$$

\section{DISCUSSION OF CALCULATIONS AND RESULTS}

\section{A. Phonon Spectra}

The construction of the energy-wave-number characteristic $F(q)$ is the most critical part of any first-principles calculation of the dispersion relations from pseudopotential theory. Its low- $q$ values strongly influence the longitudinal modes as $Q / Q_{\max }$ goes to zero. To avoid a poorly behaved LA branch, this means particular care must be given to the numerical techniques used in constructing $F(q)$ for low- $q$ values. A plot of $F(q)$ for intermediate values of $q$ is shown in Fig. 2. Selected values of this function, which was constructed from the $1 s$ HFS wave function ${ }^{5}$ for $\mathrm{Be}^{++}$with the modified dielectric function $\epsilon^{*}(q)$, are tabulated in Table IV. When the band-structure components for the dynamical matrix are formed by summing over reciprocal-lattice vectors, an interpolation scheme must be used for values of $F(q)$ intermediate to those tabulated. In the low $-q$ region, the graph of $q$ versus $F(q)$ is a straight line when plotted on log paper. This indicates that the most accurate interpolation for the low $-q$ region is obtained by using an equa- 


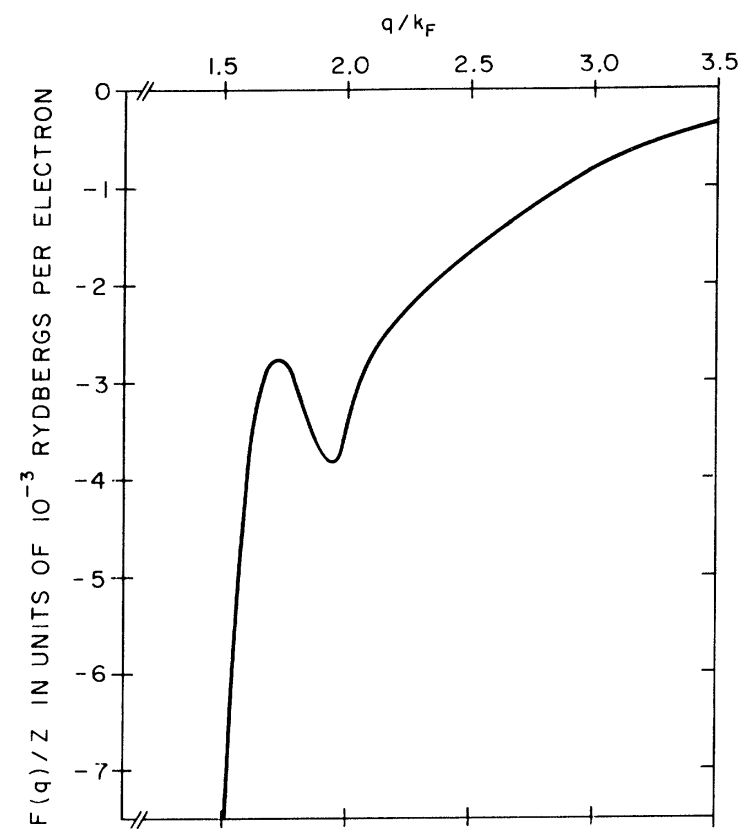

FIG. 2. Energy-wave-number characteristic for beryllium constructed from the HFS wave function for $\mathrm{Be}^{++}$.

tion of the form $y=a+b x^{-2}$. Above $q / k_{F} \simeq 0.8$, the best interpolation resulted from Lagrange's formula for a fourth-degree polynomial.

The Coulomb components of the dynamical matrix were evaluated by summing over 100 vectors in direct and reciprocal space. Convergence was very rapid, and 50 or 60 vectors in each space would have sufficed. The electronic or band-structure components are constructed entirely in $q$ space and the sums were carried out to 1533 reciprocallattice vectors. The largest elements are those from which the longitudinal branches are calculated and these elements had converged to four decimal places at 1533 vectors.

The theoretical dispersion relations for the [0001], the $[01 \overline{1} 0]$, and the $[11 \overline{2} 0]$ directions are shown in Fig. 1. The experimental points are from neutronscattering measurements by Schmunk. ${ }^{39}$ The dashed curve results from the $F(q)$ constructed with the free-electron dielectric function $\epsilon(q)$ while the solid curves are from the $F(q)$ incorporating the modified dielectric function $\epsilon^{*}(q)$. As can be seen from Fig. 1 , only the LO branch is modified sufficiently to show up on the scale of the drawing. Exactly analogous results were found in a calculation of the magnesium phonon spectrum using the same pseudopotential formalism. ${ }^{40}$ This suggests that within the approximation adopted for the dielectric screening function, the results for the calculated phonon spectrum are relatively insensitive to the choice of exchange approximation.

An earlier calculation ${ }^{14}$ of these dispersion relations made use of an analytic wave function for $\mathrm{Be}^{++}$from Green et al. ${ }^{41}$ and the results for the [0001] direction are shown in Fig. 3 for comparison with the HFS results. In addition to the slightly different fit to the experimental data, a hump near $Q / Q_{\max }=0.6$ in the TA branch does not appear in the curves constructed from the HFS wave function. Although over 1700 reciprocal-lattice vectors were summed over for the results in Fig. 3, the small splittings at the zone boundary indicate that the sums had not yet fully converged. The potential due to the orthogonalization hole was varied in the calculation using the analytic wave function and for the results shown $V_{10}$ had the value 7.6242 .

We assumed in Secs. I and III that the core-core repulsion in beryllium is small and was, therefore, neglected. There is, however, some indication that the core repulsion is not negligible in beryllium. Joshi and Rajagopal ${ }^{15}$ have pointed out that analysis of neutron data for beryllium indicates the presence of considerable trace-variable forces which they assume arises partly from the ion-core repulsion.

TABLE IV. Energy-wave-number characteristic for beryllium in units of rydbergs per electron, calculated with the modified dielectric function $\epsilon^{*}(q)$.

\begin{tabular}{|c|c|c|c|c|c|}
\hline$q / k_{F}$ & $F(q) / Z$ & & $q / k_{F}$ & $F(q) / Z$ & \\
\hline 0.01 & -0.496299 & $E 4$ & 2.10 & -0.274434 & $E-2$ \\
\hline 0.03 & -0.550926 & $E 3$ & 2.20 & -0.244570 & $E-2$ \\
\hline 0.05 & -0.197961 & $E 3$ & 2.30 & -0.218854 & $E-2$ \\
\hline 0.07 & -0.100717 & $E 3$ & 2.40 & -0.195000 & $E-2$ \\
\hline 0.09 & -0.606991 & $E 2$ & 2.50 & -0.172506 & $E-2$ \\
\hline 0.10 & -0.490568 & $E 2$ & 2.60 & -0.151423 & $E-2$ \\
\hline 0.20 & -0.118399 & $E 2$ & 2.70 & -0.131920 & $E-2$ \\
\hline 0.30 & -0.496198 & $E 1$ & 2.80 & -0.114136 & $E-2$ \\
\hline 0.40 & -0.257028 & $E 1$ & 2.90 & -0.981355 & $E-3$ \\
\hline 0.50 & -0.147881 & $E 1$ & 3.00 & -0.839101 & $E-2$ \\
\hline 0.60 & -0.900568 & $E 0$ & 3.10 & -0.713932 & $E-3$ \\
\hline 0.70 & -0.565192 & $E 0$ & 3.20 & -0.604774 & $E-3$ \\
\hline 0.80 & -0.359263 & $E 0$ & 3.30 & -0.510308 & $E-3$ \\
\hline 0.90 & -0.228297 & $E 0$ & 3.40 & -0.429097 & $E-3$ \\
\hline 1.00 & -0.143363 & $E 0$ & 3.50 & -0.359679 & $E-3$ \\
\hline 1.10 & -0.879708 & $E-1$ & 3.60 & -0.300637 & $E-3$ \\
\hline 1.20 & -0.521097 & $E-1$ & 3.70 & -0.250638 & $E-3$ \\
\hline 1.30 & -0.294075 & $E-1$ & 3.80 & -0.208458 & $E-3$ \\
\hline 1.40 & -0.156322 & $E-1$ & 3.90 & -0.172995 & $E-3$ \\
\hline 1.50 & -0.788919 & $E-2$ & 4.00 & -0.143269 & $E-3$ \\
\hline 1.60 & -0.413246 & $E-2$ & 4.50 & -0.543113 & $E-4$ \\
\hline 1.70 & -0.287870 & $E-2$ & 5.00 & -0.198442 & $E-4$ \\
\hline 1.75 & -0.283209 & $E-2$ & 5.50 & -0.716111 & $E-5$ \\
\hline 1.80 & -0.301345 & $E-2$ & 6.00 & -0.278150 & $E-5$ \\
\hline 1.90 & -0.362625 & $E-2$ & 7.00 & -0.103692 & $E-5$ \\
\hline 1.95 & -0.381423 & $E-2$ & 8.00 & -0.920454 & $E-6$ \\
\hline 2.00 & -0.353704 & $E-2$ & 10.00 & -0.642702 & $E-6$ \\
\hline
\end{tabular}




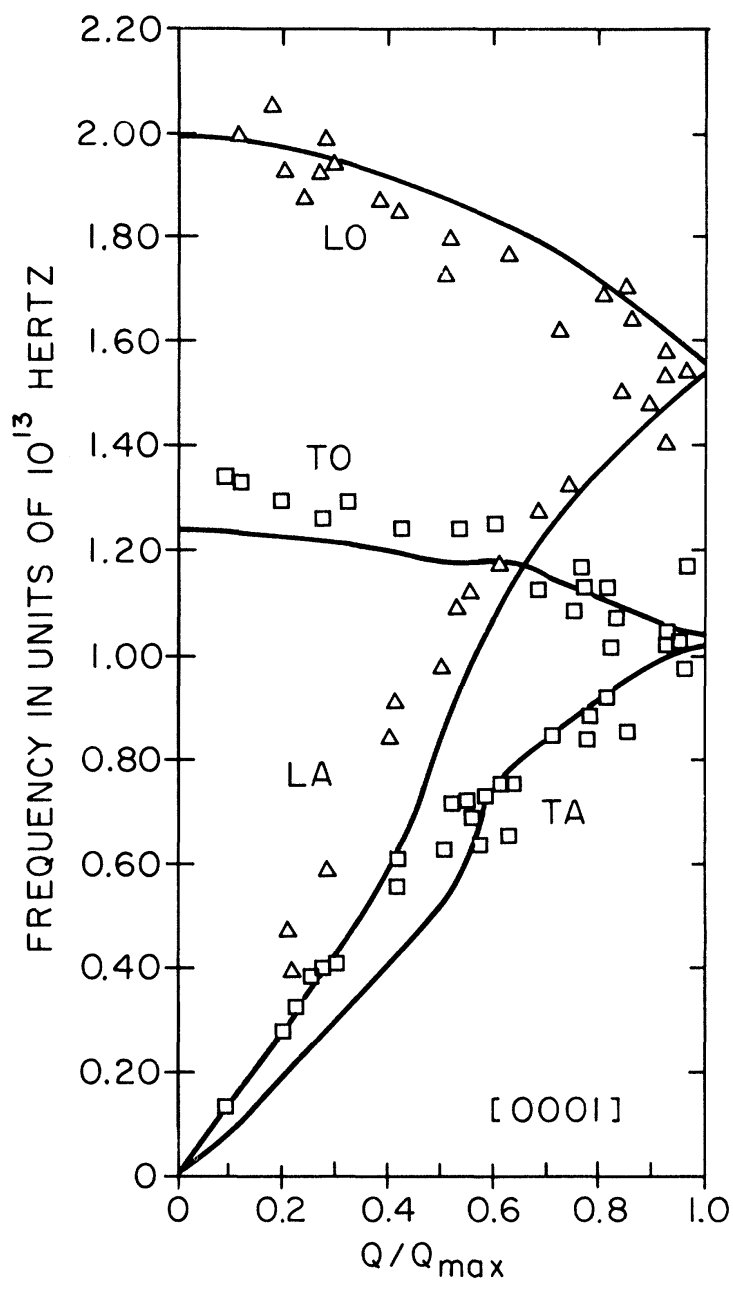

FIG.3. Theoretical curves for the [0001] direction. An analytic wave function for $\mathrm{Be}^{++}$from Green et al. (Ref. 41) was used to construct the pseudopotential for these curves. Experimental points are from Schmunk (Ref. 39).

One method for including this interaction is by using a Born-Mayer potential ${ }^{42}$ of the form

$$
U(r)=b \exp \left[\left(2 r_{1}-r\right) / \rho\right],
$$

where the parameters $b, r_{1}$, and $\rho$ are to be determined. Czachor ${ }^{43}$ used a potential of this form in his work on the lattice dynamics of hexagonal metals and he chose the parameters to be essentially those found by Born and Mayer ${ }^{44}$ for alkalis. When this potential with Czachor's parameters is included in the present calculation, the acoustical branches of the resulting dispersion relations are raised slightly, thus, somewhat improving the comparison with experiment. It is, however, not immediately obvious that the inclusion of the Born-Mayer potential with these parameters is justifiable. Vosko ${ }^{45}$ has pointed out that the extrapolation of the parameters from the theory of ionic crystals is not valid for metals and results in factors which are much too large. Since the treatment of core-core repulsion is by the introduction of an ad hoc potential and since it is also assumed to be a very small contribution in beryllium, ${ }^{34}$ we have not included this ad hoc correction in this calculation.

\section{B. Elastic Shear Constants}

The elastic shear constants were computed by summing over the same number of direct- and reciprocal-space vectors as in the evaluation of the dynamical matrix. Previously published results ${ }^{46}$ for these shear constants were obtained with a faulty transformation matrix and the numerical values are in error. ${ }^{47}$

The calculation of the band-structure contributions to the shear constants requires the first and second derivatives of $F(q)$ with respect to $q$. As we only have the tabular form of this critical function, the evaluation of the derivatives is inherently plagued with difficulties and the resulting electronic contributions may contain errors that are impossible to estimate. The procedure we used was to piecewise fit a second-order polynomial of the form $y$ $=a+b x^{2}$ to neighboring points of $F(q)$ and use the derivatives of the polynomial. At high $q$ values we used an equation of the form $y=a+b x^{-2}$ as this function better interpolates $F(q)$ at both high and low $q$ values. The results are listed in Table $\mathrm{V}$ along with experimental values from Smith and Arbogast. ${ }^{48}$ On the basis of these calculations, the electrostatic energy of the ion cores predominantly determines the elastic shear constants $C$ and $C^{\prime}$, while the results for $c_{44}$ indicate that nonelectrostatic forces, possibly ion-core repulsive interactions, are responsible for about $40 \%$ of this shear constant.

\section{ACKNOWLEDGMENTS}

The authors would like to express their appreciation to the staffs of the computation centers at The Pennsylvania State University and The Technical University of Denmark for their cooperation

TABLE V. Contributions to the elastic shear constants of beryllium in units of $10^{12} \mathrm{dyn} / \mathrm{cm}^{2}$. Experimental values are from Smith and Arbogast (Ref. 48).

\begin{tabular}{lrrr}
\hline \hline Contribution & $C$ & \multicolumn{1}{c}{$C^{\prime}$} & \multicolumn{1}{c}{$c_{44}$} \\
\hline Electrostatic & 10.3740 & 1.3227 & 1.0204 \\
Electronic & -0.7452 & -0.0066 & -0.0929 \\
Total & 9.6288 & 1.3161 & 0.9275 \\
Experimental & 9.358 & 1.329 & 1.625 \\
\hline \hline
\end{tabular}


and assistance. One of us (W. F. K. ) would like to thank Professor V. Frank and Professor A. R. Mackintosh for their assistance and hospitality

*Work based in part on a dissertation submitted by Walter F. King, III, in partial fulfillment of the requirements for the $\mathrm{Ph}$. D. in the Department of Physics at The Pennsylvania State University, University Park, Pa. 16802 .

†Research sponsored by U. S. Air Force Office of Scientific Research, Office of Aerospace Research, under AFOSR Grant No. 69-1704.

${ }^{1}$ W. A. Harrison, Phys. Rev. 136, A1107 (1964).

${ }^{2}$ L. J. Sham, Proc. Roy. Soc. (London) A283, 33 (1965).

${ }^{3}$ J. C. Phillips and L.Kleinman, Phys. Rev. 116, 287 (1959).

${ }^{4}$ W. A. Harrison, Phys. Rev. 129, 2503 (1963); 129, 2512 (1963)。

${ }^{5} \mathrm{~F}$. Herman and S. Skillman, Atomic Structure Calculations (Prentice-Hall, Englewood Cliffs, N. J., 1963).

${ }^{6}$ V. C.Sahni, G。 Venkataraman, and A. P. Roy, Phys. Letters $\underline{23}, 633$ (1966)。

${ }^{7} \mathrm{~J}$. U. Koppel and A. A. Maradudin, Phys. Letters 24A, 244 (1967).

${ }^{8}$ A. P. Roy and G. Venkataraman, Phys. Rev. 156 , 769 (1967)

${ }^{9}$ V. C. Sahni and G. Venkataraman, Phys. Rev. $\underline{185}$, 1002 (1969).

${ }^{10}$ T. L. Loucks and P. H. Cutler, Phys. Rev. 133, A819 (1964).

${ }^{11} \mathrm{~V}$. Heine and I. V. Abarenkov, Phil. Mag. 9, 451 (1964) .

${ }^{12}$ G. Gilat, G. Rizzi, and G. Cubiotti, Phys. Rev. 185, 971 (1969)

${ }^{13}$ R.W. Shaw, Jr., and W. A. Harrison, Phys. Rev. 163,604 (1967).

${ }^{14}$ W. F. King and P. H. Cutler, Solid State Commun. 7, 295 (1969).

${ }^{15} \mathrm{~S}$. K. Joshi and A. K. Rajagopal, Solid State Physics, edited by F. Seitz, D. Turnbull, and H. Ehrenreich, (Academic, New York, 1968), Vol. 22 。

${ }^{16} \mathrm{~W}$. A. Harrison, Pseudopotentials in the Theory of Metals (Benjamin, New York, 1966).

${ }^{17}$ P. P. Ewald, Ann. Phys. Leipzig 64, 253 (1921).

${ }^{18} \mathrm{M}$. Born and K. Huang, Dynamical Theory of Crystal Lattices (Oxford U. P. , London, 1954).

${ }^{19}$ P. J. Lin and J. C. Phillips, Advan. Phys. 14, 257 (1965)。 during his stay at The Technical University of Denmark, during which time some of these calculations were performed.
${ }^{20} \mathrm{~W}$. A. Harrison, Ref. 16, Chap. 8.

${ }^{21}$ R. Pick and G. Sarma, Phys。Rev. 135, A1363 (1964)

${ }^{22}$ J. H. C. Thompson, Proc. Roy. Soc. (London) A149, 487 (1935)。

${ }^{23}$ W $。$ F。 King, Phys。 Letters 27A, 386 (1968).

${ }^{24}$ W. Cochran, in Phonons in Perfect Lattices and in Lattices with Point Imperfections, edited by R. W. H. Stevenson (Oliver and Boyd, London, 1966).

${ }^{25}$ W. A. Harrison, Ref. 16, Chap. 7.

${ }^{26} \mathrm{D}$. R. Hartree, The Calculation of Atomic Structures (Wiley, New York, 1957).

${ }^{27}$ W. Kohn and L. J. Sham, Phys. Rev. 140, A1133 (1965).

${ }^{28}$ J. C. Slater, Phys. Rev. 81, 385 (1951).

${ }^{29}$ S. Golin, Phys. Rev. 140, A993 (1965).

${ }^{30}$ D. Pines, Elementary Excitations in Solids (Ben-

jamin, New York, 1964).

${ }^{31} \mathrm{M}$. H. Cohen and J. C. Phillips, Phys.' Rev. 124, 1818 (1961).

${ }^{32}$ J. Hubbard, Proc. Roy. Soc. (London) A240, 539

(1957); A243, 336 (1958).

${ }^{33}$ D. Pines, Ref. 30, p. 239

${ }^{34}$ J. U. Koppel, Ph. D. thesis, University of California at San Diego, 1968 (unpublished).

${ }^{35}$ J. R. Reitz and C. S. Smith, Phys. Rev. 104, 1253 (1956)。

${ }^{36}$ C. S. G. Cousins, J. Phys. C 1,478 (1968).

${ }^{37}$ H. B. Huntington, Phys. Rev. 57,60 (1940).

${ }^{38}$ K. Fuchs, Proc. Roy. Soc. (London) A153, 622 (1936).

${ }^{39}$ R. E. Schmunk, Phys. Rev. 149, 450 (1966).

${ }^{40}$ W. F. King and P. H. Cutler (unpublished).

${ }^{41}$ L. C. Green, M. M. Mulder, M. N. Lewis, and

J. W. Woll, Phys。Rev. 93, 757 (1954).

${ }^{42} \mathrm{~F}$. Seitz, The Modern Theory of Solids (McGrawHill, New York, 1940).

${ }^{43}$ A. Czachor, Phys. Status Solidi 29, 423 (1968).

${ }^{44}$ M. Born and J. Mayer, Z. Physik 75, 1 (1932).

${ }^{45}$ S. H. Vosko, Phys. Letters 13,97 (1964).

${ }^{46}$ W. F. King, III and P. H. Cutler, Phys. Letters 28A, 289 (1968).

${ }^{47}$ The authors are indebted to Dr. C. S. G. Cousins for pointing out the source of this mistake.

${ }^{48}$ J. F. Smith and C. L. Arbogast, J. Appl. Phys. 31, 99 (1960). 